\title{
MicroRNA-2053 overexpression inhibits the development and progression of hepatocellular carcinoma
}

\author{
TAO SONG ${ }^{1}, \mathrm{KE} \mathrm{MA}^{2}, \mathrm{CUI} \mathrm{ZHAO}^{3}$, JIJIN YANG $^{4}$ and JINGYU LIU ${ }^{4}$ \\ ${ }^{1}$ Department of Medical Imaging, Changhai Hospital of Second Military Medical University of Chinese PLA, \\ Shanghai 200433; ${ }^{2}$ Shandong Co-Innovation Center of Classic TCM Formula, Shandong University of \\ Traditional Chinese Medicine, Jinan, Shandong 250355; ${ }^{3}$ Department of General Medicine, Affiliated \\ Hospital of Chengde Medical College, Chengde, Hebei 067000; ${ }^{4}$ Department of Interventional Radiology, \\ Changhai Hospital of Second Military Medical University of Chinese PLA, Shanghai 200433, P.R. China
}

Received November 28, 2017; Accepted February 4, 2019

DOI: $10.3892 / 01.2019 .10501$

\begin{abstract}
MicroRNAs (miRNAs) represent a class of small RNAs that participate in the regulation of tumor progression. However, the identification of functional miRNAs in tumors has not been thoroughly elucidated. In the present study we aim to investigate the impact of altered miR-2053 expression in hepatocellular carcinoma (HCC) cells. The results of the present study demonstrated that miR-2053 overexpression inhibited cell proliferation, migration and invasion, and promoted apoptosis in a HCC cell line, while miR-2053 knockdown induced the opposite cellular phenotypic changes. Mechanistically, it was found that overexpression of miR-2053 resulted in the downregulation of the phosphoinositide 3 -kinase $(\mathrm{PI} 3 \mathrm{~K})$ and $\mathrm{Wnt} / \beta$-catenin signaling pathways, which are aberrantly expressed in HCC. Collectively, the results indicate that miR-2053 serves as a tumor suppressor with a crucial role in inhibiting the proliferation, migration and invasion of $\mathrm{HCC}$ via targeting the PI3K and Wnt/ $\beta$-catenin signaling pathways. These data indicate a potential application of miR-2053 in cancer therapy.
\end{abstract}

\section{Introduction}

Hepatocellular carcinoma (HCC) accounts for up to $90 \%$ of all primary liver cancer worldwide, and has been reported to cause $>662,000$ mortality cases worldwide annually, particularly in developing countries $(1,2)$. Despite improvements in the treatment of liver cancer, the survival rate after 5 years

Correspondence to: Professor Jijin Yang or Dr Jingyu Liu, Department of Interventional Radiology, Changhai Hospital of Second Military Medical University of Chinese PLA, 168 Changhai Road, Yangpu, Shanghai 200433, P.R. China

E-mail: jijinyang@sina.com

E-mail: liujingyu1603@163.com

Key words: microRNA-2053, proliferation, migration, invasion, phosphoinositide 3-kinase, Wnt is $<30 \%$ due to its high recurrence and metastasis rate $(3,4)$. Although the treatment efficacy for HCC is improving, the lack of biomarkers for early diagnosis and effective therapeutic targets results in consistently poor curative rates. Consequently, a better understanding of the mechanisms and potential biomarkers are warranted to improve HCC patient treatment.

MicroRNAs (miRNAs) are small, single-stranded, non-coding functional RNA molecules of $\sim 22$ nucleotides that are widely present in eukaryotic cells $(5,6)$. MiRNAs have been identified in various cancer types and are involved in cancer development and progression, acting as oncogenes or tumor suppressors $(7,8)$. Previous studies show that miRNAs are involved in liver disease and progression of liver cancer. For example, miR-324-3p promotes HCC growth, however, miR-148b serves as a tumor suppressor in HCC by inhibiting proliferation and invasion (9). In addition, several miRNA signatures are related to chronic hepatic infection, cirrhosis, and steatosis $(10,11)$. The specific expression of certain miRNAs has been found to have biological behavior in terms of tumor aggressiveness, metastatic potential and even responsiveness to treatment. Therefore, any abnormal expression of miRNA molecules is thought to be associated with hepatocarcinogenesis. Accumulating evidence suggests that multiple miRNAs are aberrantly expressed in and associated with various processes of cellular carcinoma development. miR-2053 is a miRNA molecule located at 8q23.3 in the human genome. It has been reported that miR-2053 may induce proliferation of adult cardiomyocytes (12). However, to the best of our knowledge, the function of miR-2053 in HCC has not been explored.

Hepatocarcinogenesis is a complex, multistep process in which several signaling cascades are altered, leading to the development of a heterogeneous biological tumor. The phosphoinositide 3-kinase (PI3K)/serine/threonine protein kinase (AKT)/Mammalian target of rapamycin (mTOR) and the Wnt/ $\beta$-catenin pathways serve important roles in HCC proliferation and cell cycle progression $(13,14)$. Studies have shown that PI3K signaling is activated in $30-50 \%$ of HCC cases, and the downstream ribosomal protein S6 (RPS6) is also activated in $50 \%$ of HCC patients (15). Previous studies have demonstrated that the $\mathrm{Wnt} / \beta$-catenin signaling pathway 
plays a critical role in proliferation and cell cycle progression of HCC cells $(16,17)$. miR-1247-5p functions as a tumor suppressor by activating Wnt3 (18). It was also demonstrated that miR-138 modulates prostate cancer cell invasion and migration via the $\mathrm{Wnt} / \beta$-catenin pathway (19). In the present study, the association of miR-2053 with the PI3K/AKT/mTOR and $\mathrm{Wnt} / \beta$-catenin pathways in HCC cells was explored.

The effects and mechanisms of miR-2053 on the progression of HCC were investigated. The data demonstrated that overexpression of miR-2053 inhibited the proliferation, migration and invasion of HCC cells and induced cell apoptosis, which may be through inhibiting the activation of the AKT/mTOR and Wnt/ $\beta$-catenin pathways. These findings reveal the function and regulatory mechanisms of miR-2053 in human HCC.

\section{Materials and methods}

Cell lines and cell culture. The human HCC cell line Huh7 was obtained from Cell Bank of Chinese Academy of Sciences (Shanghai, China) and routinely maintained in high-glucose Dulbecco's Modified Eagle's Media (DMEM) supplemented with $10 \%$ fetal bovine serum (FBS; Gibco; Thermo Fisher Scientific, Inc., Waltham, MA, USA), $100 \mathrm{U} / \mathrm{ml}$ penicillin, and $100 \mathrm{mg} / \mathrm{ml}$ streptomycin. All cell lines were cultured at $37^{\circ} \mathrm{C}$ in a humidified atmosphere containing $5 \% \mathrm{CO}_{2}$.

Plasmid transfection. Cells were seeded in a 24-well plate and after the cells reached $80 \%$ confluence, transient transfection of miRNA plasmids was carried out using Lipofectamine ${ }^{\circledR}$ 2000 (Invitrogen; Thermo Fisher Scientific, Inc.), according to the manufacturer's protocol. Briefly, the miR-2053 or siRNA-miR-2053 sequences were cloned into the pCMV-MIR vectors (Guangzhou Ribobio Co.,Ltd., Guangzhou, China) (20). Lipofectamine $2000(10 \mu \mathrm{l})$ was added to $250 \mu \mathrm{l}$ DMEM without serum and incubated for $5 \mathrm{~min}$ at room temperature. The plasmids [pCMV-MIR-miR-2053 (miR-2053) or pCMV-MIR-si-miR-2053 (si-miR-2053), $50 \mathrm{nM}$ ] were added to $250 \mu \mathrm{l}$ DMEM without serum. After $5 \mathrm{~min}$ incubation, complexes were formed by the total of the two dilutions as aforementioned, gently mixing and incubating for $30 \mathrm{~min}$ at room temperature. The cells were prepared by removing medium and washing twice with PBS followed by addition of $0.5 \mathrm{ml}$ of medium without serum. The complexes $(500 \mu \mathrm{l})$ were added to each well, and the plate was mixed gently by rocking back and forth, and incubated at $37^{\circ} \mathrm{C}$ in a humidified atmosphere containing $5 \% \mathrm{CO}_{2}$ for $24 \mathrm{~h}$ prior to evaluating gene expression. The medium was changed $6 \mathrm{~h}$ after transfection. Cells transfected with an empty pCMV-MIR vector were used as a negative control group (pNC) for the miR-2053 upregulation group, cells transfected with pCMV-MIR-siRNA (non-coding siRNA) vector was performed as a negative control (siNC) for si-miR-2053, and cells without any treatment was used as blank control group (Con). The mature sequence of miR-2053 is 5'-GUGUUAAUUAAACCUCUA UUUAC-3'; the siRNA targeting miR-2053 is 5'-GUAAAU AGAGGUUUAAUUAACAC-3'.

RNA isolation and reverse transcription-quantitative polymerase chain reaction $(R T-q P C R)$. Total RNA was extracted using an RNA extraction kit (CoWin Bioscience Co., Ltd., Beijing, China) and total RNA was converted to cDNA using a miRNA cDNA Synthesis Kit (CoWin Bioscience Co., Ltd.). miRNA levels were examined using a miRNA qPCR detection kit (CoWin Bioscience Co., Ltd.). The thermocycling conditions were set at $95^{\circ} \mathrm{C}$ for $5 \mathrm{~min}$, followed by 40 cycles of $95^{\circ} \mathrm{C}$ for $10 \mathrm{sec}, 60^{\circ} \mathrm{C}$ for $30 \mathrm{sec}$, and $72^{\circ} \mathrm{C}$ for $1 \mathrm{sec}$. Each reaction was performed in triplicate. The expression of miR-2053 was calculated using the $2^{-\Delta \Delta \mathrm{Cq}}$ method (21). Three independent experiments were performed to analyze the relative gene expression. The primers were as follows: MiR-2053, forward primer, 5'-GUGUUAAUUAAACCUCUAUUUAC-3'; reverse primer was obtained from the miRNA qPCR detection kit; and U6, forward primer, 5'-CTCGCTTCGGCAGCACA-3'; reverse primer, 5'-AACGCTTCACGAATTTGCGT-3'.

Western blot analysis. After transfection for $48 \mathrm{~h}$, total cellular protein was extracted using radioimmunoprecipitation (RIPA) lysis buffer (CoWin Biosciences Co., Ltd.). The lysates containing equal amounts of protein $(20 \mu \mathrm{g})$ were loaded onto $10 \%$ SDS-PAGE gels (Beijing Solarbio Science and Technology Co., Ltd., Beijing, China) and transferred onto polyvinylidene fluoride membranes (EMD Millipore, Billerica, MA, USA). The membrane was blocked with 5\% skimmed milk for $1 \mathrm{~h}$ and incubated with primary antibodies overnight at $4^{\circ} \mathrm{C}$, followed by $1 \mathrm{~h}$ of incubation with horseradish peroxidase-conjugated secondary antibodies (anti-rabbit IgG, cat. no. SA00001-2; anti-mouse IgG, cat. no. SA00001-1; both 1:5,000; both ProteinTech Group, Inc., Chicago, IL, USA). Finally, the proteins were detected by enhanced chemiluminescence using the ECL Plus kit (ProteinTech Group, Inc.). Band densities were analyzed using the QUANTITY ONE 1-D software (version 4.6.7; Bio-Rad Laboratories, Inc., Hercules, CA, USA). The following primary antibodies were used for analysis: Anti-caspase-9 (cat. no. ab219590; 1:1,000), anti-AKT (cat. no. ab32505, 1:1,000), anti-phosphorylated AKT (cat.no.ab81283, 1:1,000), anti-mTOR (cat.no.ab2732, 1:1,000), anti-phosphorylated mTOR (cat.no.ab131538, 1:100), anti-Wnt3 (cat. no. ab32249, 1:10), anti- $\beta$-catenin (cat. no. ab32572, 1:1,000, all Abcam, Cambridge, UK), anti-E-cadherin (cat. no. 3195; 1:1,000; Cell Signaling Technology, Danvers, MA, USA), anti-Cyclin D1 (cat. no. 60186-1-Ig, 1:1,000), anti-p70 (cat. no. 14485-1-AP, 1:1,000), anti-B-cell lymphoma 2 (BCL2; cat. no. 60178-1-Ig, 1:1,000), anti-BCL-2-associated X protein (BAX; cat. no. 60267-1-Ig, 1:1,000) and anti-cleaved caspase-3 (cat. no. 25546-1-AP, 1:1,000) and GAPDH (cat. no. 60004-1-Ig, 1:5,000; all Proteintech Group, Inc.), which served as the loading control.

Cell proliferation assay. After $24 \mathrm{~h}$ of transfection, the cells $\left(1 \times 10^{4}\right.$ cells $\left./ \mathrm{ml}\right)$ were plated onto 96 -well plates in $100 \mu \mathrm{l}$ of complete medium. Cell Counting Kit-8 (CCK-8; Solarbio Science \& Technology Co., Ltd., Beijing, China) was used according to the manufacturer's protocol. The plates were incubated at $37^{\circ} \mathrm{C}$ for $1.5 \mathrm{~h}$, and the absorbance at $450 \mathrm{~nm}$ was measured. The proliferation rates were determined at $0,24,48$ and $72 \mathrm{~h}$ post-transfection.

Clone formation assay. After $24 \mathrm{~h}$ of transfection, cells $(\sim 500)$ were planted in a $6-\mathrm{cm}$ dish in $5 \mathrm{ml}$ of medium and cultured 

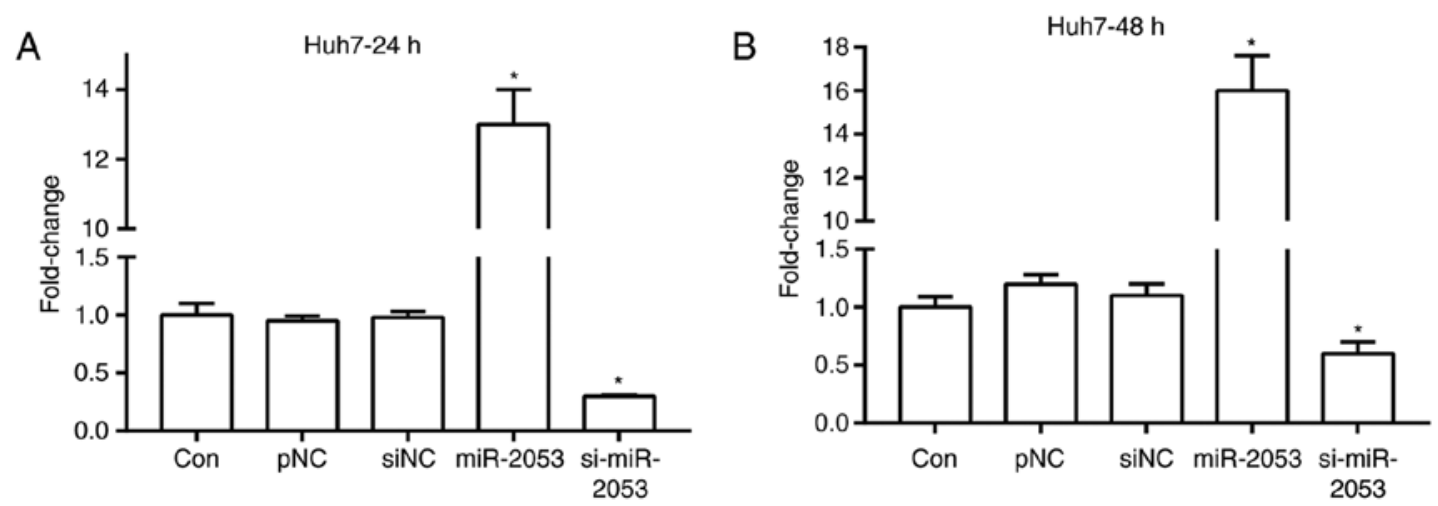

Figure 1. Identification of expression of miR-2053 in Huh7 cells. (A) Expression of miR-2053 $24 \mathrm{~h}$ after transfection. (B) Expression of miR-2053 $48 \mathrm{~h}$ after transfection. "P<0.05, miR-2053 group vs. pNC group, si-miR-2053 group vs. siNC group; $\mathrm{n}=3$. Con, untransfected group; pNC, cells transfected with pCMV-MIR empty vector; miR-2053, cells transfected with pCMV-MIR- miR-2053 vector, miR-2053 overexpression group; siNC, cells transfected with pCMV-MIR-siRNA (non-coding siRNA) vector; si-miR-2053, cells transfected with pCMV-MIR-si-miR-2053, miR-2053 knockdown group.

until visible clones formed. After staining with $0.1 \%$ crystal violet for $30 \mathrm{~min}$ at room temperature, the colonies were counted under a microscope (magnification $\mathrm{x} 4$; Nikon Corporation, Tokyo, Japan) and imaged with a camera.

Cell migration and invasion assay. Cell invasion and migration assays were performed $24 \mathrm{~h}$ after transfection using a Transwell system (EMD Millipore, Billerica, MA, USA). Migration was assessed using uncoated transwells, and invasion was investigated using transwells coated with Matrigel (BD Biosciences, San Jose, CA, USA). A total of $1 \times 10^{5}$ cells transfected for $24 \mathrm{~h}$ in serum-free medium were added to the top chamber of the transwell. The bottom chamber was filled with medium containing $10 \%$ FBS. After $24 \mathrm{~h}$ incubating at $37^{\circ} \mathrm{C}$, the cells on the upper surface of the membrane were gently removed with a cotton swab, and the membranes were washed three times with PBS, fixed and stained with $0.1 \%$ crystal violet for $5 \mathrm{~min}$ at room temperature. The cells were imaged and counted in in 5 random fields using a light microscope (Nikon Corporation; magnification x100).

Flow cytometry analysis. Cell apoptosis was measured using the fluorescein isothiocyanate (FITC) Annexin V Apoptosis Detection Kit (4A Biotech Co., Ltd., Beijing, China), according to the manufacturer's protocol. Briefly, after transfection of the cells for $24 \mathrm{~h}$, the medium was removed and the cells were incubated with serum-free medium for $24 \mathrm{~h}$. The cells were digested using trypsin, washed with ice-cold PBS, centrifuged at $200 \mathrm{x} \mathrm{g}$ for $5 \mathrm{~min}$, washed twice with ice-cold PBS and then stained with FITC-Annexin V and propidium iodide (PI). Apoptotic cells were detected using a flow cytometer (EPICS, Xl-4; Beckman Coulter, Inc., Brea, CA, USA) and analyzed using FlowJo software (v7.6.1; FlowJo LLC, Ashland, OR, USA).

Statistical analysis. All statistical analyses were performed using SPSS 18.0 software. (SPSS, Inc., Chicago, IL, USA) The data were expressed as mean \pm standard deviation. The Student's t-test was used to determine statistically significant differences between the two groups, and one-way analysis of variance was used to determine the statistical significance across multiple groups followed by Least Significant Difference post hoc comparison. $\mathrm{P}<0.05$ was considered to indicate a statistically significant difference. All experiments were performed in triplicate.

\section{Results}

miR-2053 expression in Huh7-transfected cells. To increase or decrease miR-2053 expression, pCMV-MIR-miR-2053 and pCMV-MIR-si-miR-2053 plasmids were transfected into Huh7 cells, a human HCC cell line. RT-qPCR analysis revealed that miR-2053 expression exhibited no significant difference in control, pNC and siNC groups. miR-2053 expression was significantly increased in cells transfected with pCMV-MIR-miR-2053 plasmid (miR-2053 group) compared with that in the pNC group, and decreased by siRNA-miR-2053 (si-miR-2053 group) at 24 and $48 \mathrm{~h}$ after transfection compared with that in the siNC group (Fig. $1 \mathrm{~A}$ and $\mathrm{B} ; \mathrm{P}<0.05$ ).

miR-2053 inhibits proliferation of Huh7 cells. Proliferation serves an important role in the development of a tumor (22). CCK-8 assay was used to study the role of miR-2053 in the proliferation of Huh7 cells. The proliferation rate was measured at $72 \mathrm{~h}$. Following transfection for $72 \mathrm{~h}$, proliferation of Huh7 cells was significantly inhibited by miR-2053 overexpression in the miR-2053 group compared with that in the $\mathrm{pNC}$ group, and was promoted in the si-miR-2053 group compared with that in the siNC group (Fig. 2A). Additionally, a plate colony assay showed that cells transfected with miR-2053 formed fewer and smaller colonies compared with that in the pNC group in Huh7 cells, and si-miR-2053 increased the colony number compared with the siNC group (Fig. 2B and C). These results demonstrate that overexpression of miR-2053 inhibited the proliferative potential, and miR-2053 knockdown promoted cell proliferation of this HCC cell line.

miR-2053 inhibits Huh7 cell migration and invasion. Tumor invasion refers to the process of tumor cells destroying the surrounding normal tissue structure and detaching from the primary tumor (23). It was found that the number of cells successfully invading through a Matrigel-coated membrane was inhibited significantly in the miR-2053 group compared with that in the pNC group. Additionally, the number of invaded cells increased in the si-miR-2053 group compared 

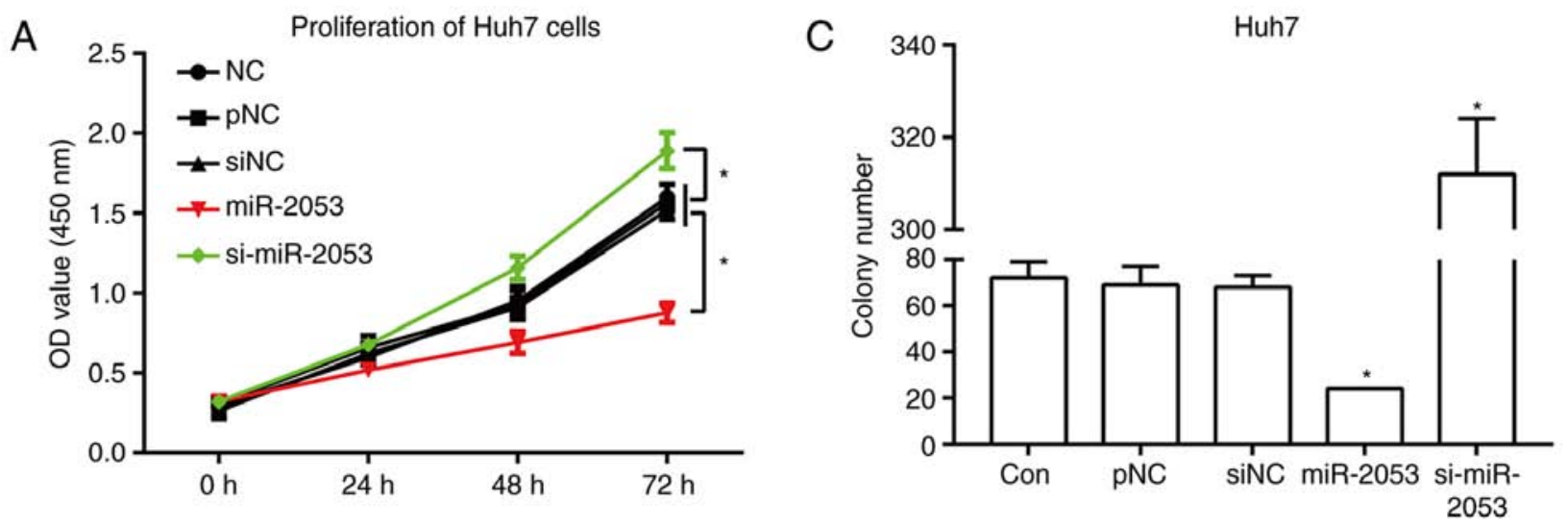

B
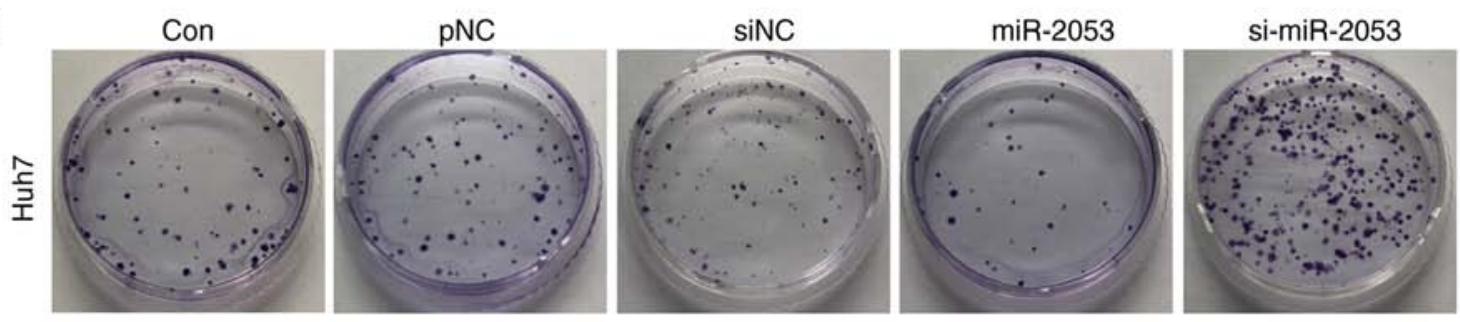

Figure 2. miR-2053 inhibits the proliferation of Huh7 cells. (A) Huh7 cell proliferation measured by Cell Counting Kit- 8 assay at $0,24,48$ and $72 \mathrm{~h}$ after transfection. (B) Representative images and (C) quantification of the number of colonies resulting from the colony formation assay, $24 \mathrm{~h}$ following transfection. ${ }^{*} \mathrm{P}<0.05$, miR-2053 group vs. pNC group, si-miR-2053 group vs. siNC group; $\mathrm{n}=3$. Con, untransfected group; pNC, cells transfected with pCMV-MIR empty vector; miR-2053, cells transfected with pCMV-MIR- miR-2053 vector, miR-2053 overexpression group; siNC, cells transfected with pCMV-MIR-siRNA (non-coding siRNA) vector; si-miR-2053, cells transfected with pCMV-MIR-si-miR-2053, miR-2053 knockdown group.

A Huh7

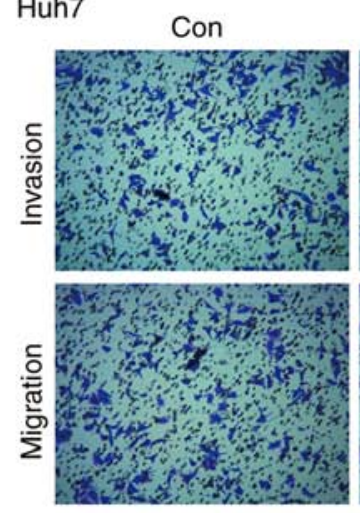

$\mathrm{pNC}$
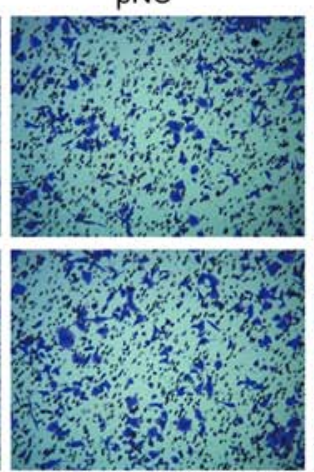

SiNC

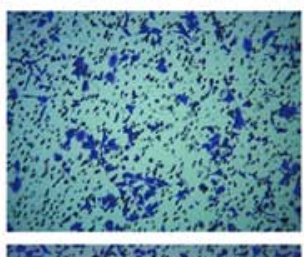

$\operatorname{miR}-2053$

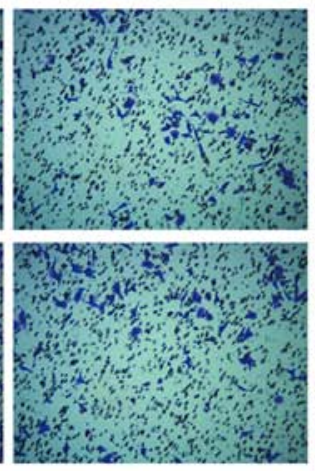

si-miR-2053

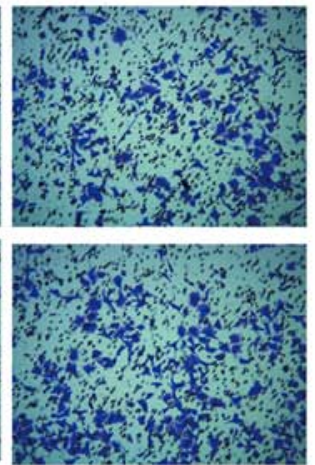

B

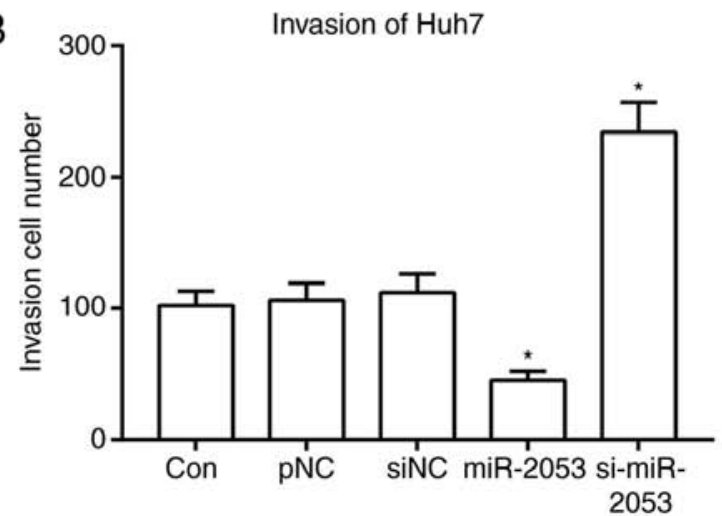

C

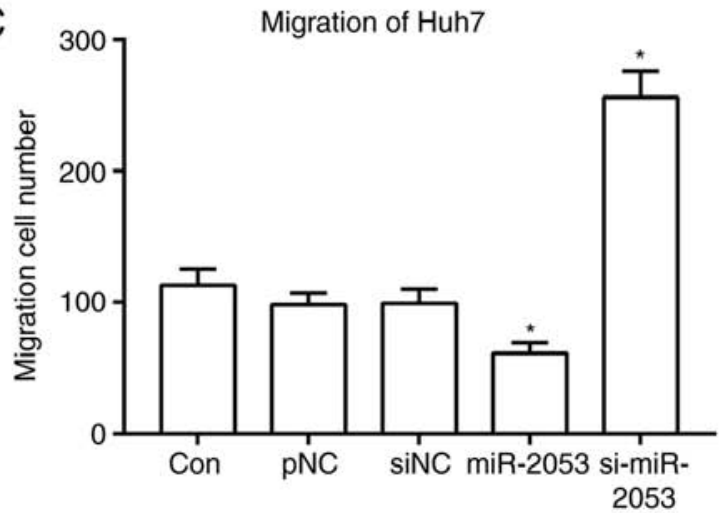

Figure 3. miR-2053 inhibits Huh7 cell migration and invasion in vitro. After $24 \mathrm{~h}$ of transfection, Transwell and Matrigel assays were performed to assess cell migration and invasion (A) Magnification, x100. Quantification of the number of (B) invasive and (C) migratory cells derived from 5 randomly chosen fields. ${ }^{*} \mathrm{P}<0.05$, miR-2053 group vs. pNC group, si-miR-2053 group vs. siNC group; $\mathrm{n}=3$. Con, untransfected group; pNC, cells transfected with pCMV-MIR empty vector; miR-2053, cells transfected with pCMV-MIR- miR-2053 vector, miR-2053 overexpression group; siNC, cells transfected with pCMV-MIR-siRNA (non-coding siRNA) vector; si-miR-2053, cells transfected with pCMV-MIR-si-miR-2053, miR-2053 knockdown group. 

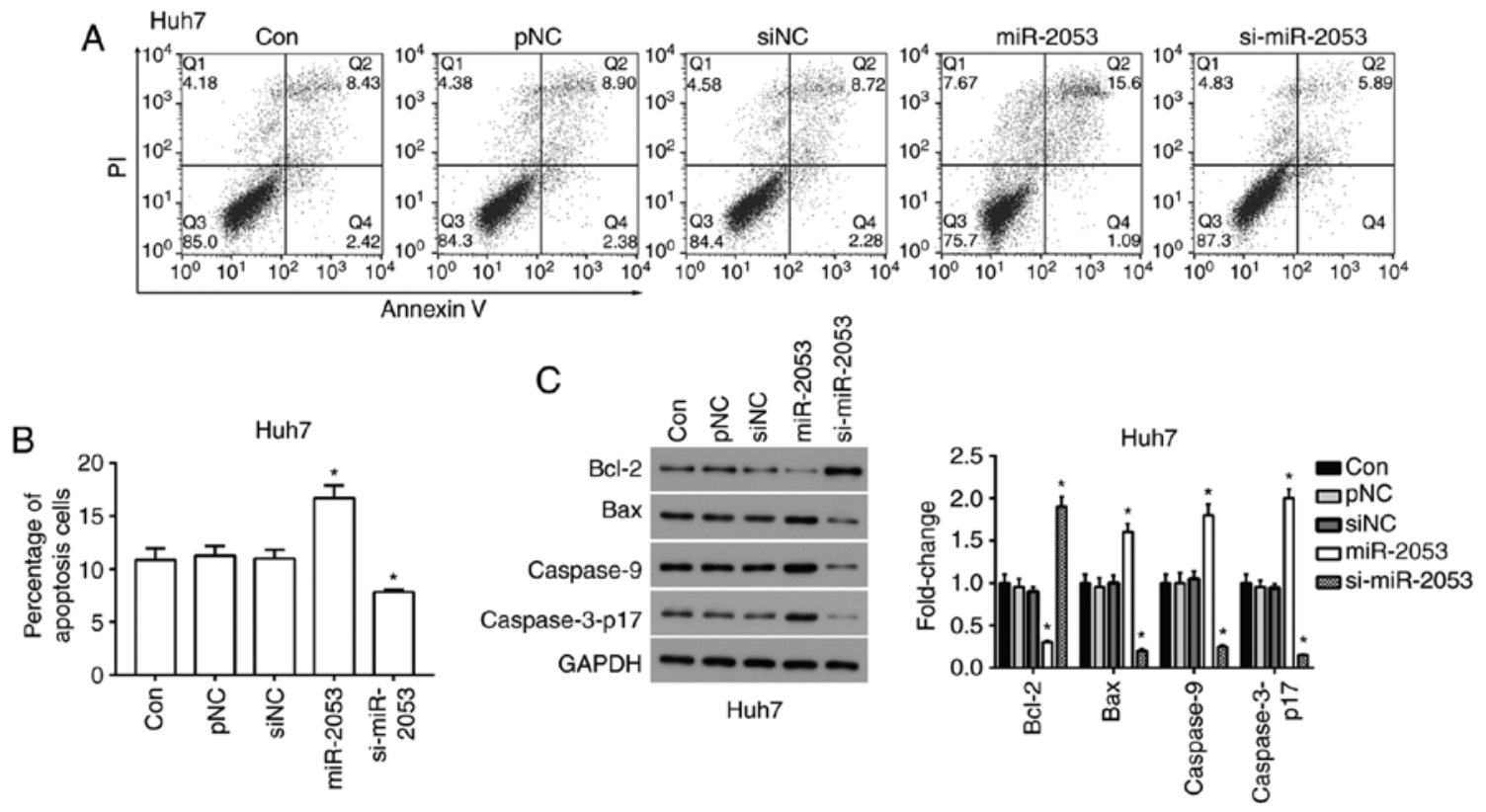

Figure 4. miR-2053 promotes apoptosis in Huh7 cells. (A) After $24 \mathrm{~h}$ of transfection, cell apoptosis was measured by flow cytometry after incubation with FITC-Annexin V and PI solution. (B) Percentage of cells positive for Annexin V and PI. (C) Protein expression levels of BCL2, BAX, caspase-9 and cleaved caspase-3. "P<0.05, miR-2053 group vs. pNC group, si-miR-2053 group vs. siNC group; $\mathrm{n}=3$. Con, untransfected group; pNC, cells transfected with pCMV-MIR empty vector; miR-2053, cells transfected with pCMV-MIR-miR-2053 vector, miR-2053 overexpression group; siNC, cells transfected with pCMV-MIR-siRNA (non-coding siRNA) vector; si-miR-2053, cells transfected with pCMV-MIR-si-miR-2053, miR-2053 knockdown group; FITC, fluorescein isothiocyanate; PI, propidium iodide; BCL2, B-cell lymphoma 2, BAX, BCL-2-associated X protein.
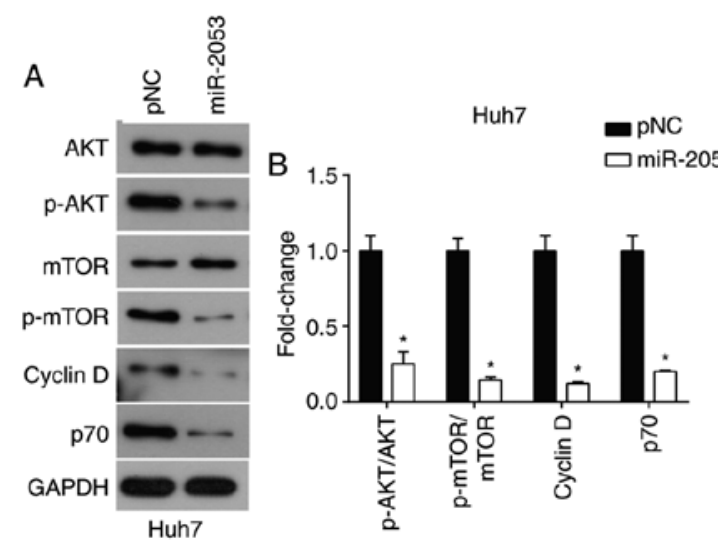

Figure 5. miR-2053 inhibits the PI3K signaling pathway. (A) Protein expression levels of total and p-AKT, total and p-mTOR, cyclin D1 and p70. (B) Ratios of $\mathrm{p}-\mathrm{AKT} / \mathrm{AKT}$ and $\mathrm{p}-\mathrm{mTOR} / \mathrm{mTOR}$, and the expression of cyclin D1 and $\mathrm{p} 70 . \mathrm{n}=3,{ }^{*} \mathrm{P}<0.05$ vs. $\mathrm{pNC}$ control group. $\mathrm{pNC}$, cells transfected with pCMV-MIR empty vector; miR-2053, cells transfected with pCMV-MIR- miR-2053 vector, miR-2053 overexpression group; p, phosphorylated; AKT, serine/threonine protein kinase; mTOR, Mammalian target of rapamycin.

with that in the siNC group (Fig. $3 \mathrm{~A}$ and $\mathrm{B} ; \mathrm{P}<0.05$ ). The number of cells migrating through non-coated membranes also decreased markedly in miR-2053-overexpresing Huh7 cells, and increased significantly in si-miR-2053 group (Fig. 3A and $\mathrm{C} ; \mathrm{P}<0.05)$. These results demonstrate that miR-2053 markedly suppresses the migration and invasion of Huh7 cells.

miR-2053 promotes apoptosis in Huh7 cells. Apoptosis plays an important role in the process of tumor growth (24). The effect of miR-2053 overexpression or knockdown on apoptosis

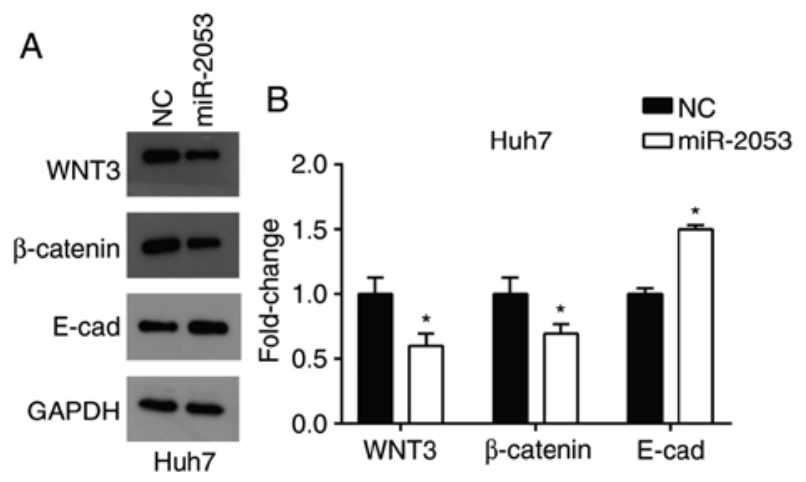

Figure 6. miR-2053 inhibits the Wnt/ $\beta$-catenin signaling pathway. (A) Protein expression levels of Wnt3, $\beta$-catenin and epithelial-cadherin. (B) Quantitative analysis of western blot results. $\mathrm{N}=3,{ }^{*} \mathrm{P}<0.05$ vs. pNC group. pNC, cells transfected with pCMV-MIR empty vector; miR-2053, cells transfected with pCMV-MIR- miR-2053 vector, miR-2053 overexpression group; E-cad, E-cadherin.

was investigated in Huh7 cells. As shown in Fig. 4, 16.69\% of cells overexpressing miR-2053 were positive for Annexin V and considered apoptotic. Of the cells transfected with si-miR-2053, 8.00\% were apoptotic, while the siNC group contained $11.00 \%$ apoptotic cells (Fig. 4A and B). The mechanisms of miR-2053-induced apoptosis was further determined by analyzing the expression of 4 apoptosis-related proteins including BCL2, BAX, caspase-9 and cleaved caspase-3 (caspase-3-p17). BCL2 is an anti-apoptotic protein and BAX is a pro-apoptotic protein, while caspase- 9 and activated caspase-3 also play key roles in the process of apoptosis $(25,26)$. The results showed that overexpression of miR-2053 downregulated the level of BCL2 and upregulated BAX, caspase-9 and 
activated caspase- 3 expression compared with that in the $\mathrm{pNC}$ group. Transfection with si-miR-2053 exhibited the opposite effect (Fig. 4C). Thus, these results strongly indicate that miR-2053 induces apoptosis in Huh7 cells through activating caspase- 3 and caspase- 9 .

miR-2053 overexpression inhibits activation of the PI3K/AKT signaling pathway in Huh7 cells. The PI3K signaling pathway serves a central role in the progression of HCC, participating in proliferation and metastasis (27). According to our previous results, it was demonstrated that miR-2053 overexpression decreased cell proliferation, migration and invasion. This effect may be mediated through PI3K signaling. Therefore, the impact of miR-2053 overexpression on the PI3K/AKT signaling pathway was assessed. It was found that the ratio of phosphorylated (p) AKT to total AKT, the ratio of p-mTOR to total mTOR, and the levels of p70 and Cyclin D1 were downregulated in the miR-2053 group (Fig. 5). These results highlight an association between miR-2053 and the PI3K/AKT signaling pathway in Huh7 cells.

miR-2053 inhibits activation of the Wnt/ $\beta$-catenin signaling pathway in Huh7 cells. Previous studies have found that miRNAs promote HCC invasion and metastasis via the Wnt/ $\beta$-catenin signaling pathway $(28,29)$. The results of the present study demonstrated that overexpression of miR-2053 resulted in a reduction in Wnt3 protein levels. It was also revealed that $\beta$-catenin was decreased and E-cadherin was increased in miR-2053-overexpressing Huh7 cells compared with that in the pNC group (Fig. 6). These results indicate that miR-2053 may impact HCC progression through the $\mathrm{Wnt} / \beta$-catenin signaling pathway.

\section{Discussion}

Hepatocellular carcinoma is the third leading cause of cancer-associated mortality and the incidence of viral infections is increasing worldwide (30). Research has shown that miRNAs are abnormally expressed in HCC, and are involved in the regulation of tumor development and malignant changes (31). Certain miRNAs serve as promoters in hepatic tumorigenesis. Conversely, several miRNAs function as tumor suppressors. For example, miR-221 blockage prompts HCC survival $(32,33)$, whereas miR-148b suppresses cell proliferation and invasion in HCC by targeting the Wnt $1 / \beta$-catenin pathway (34). Studies have found that miR-132 inhibits cell proliferation, invasion and migration of HCC (35). These findings indicate that miRNAs are intimately involved in the progression of HCC malignancy.

In the present study, it was shown that the overexpression of miR-2053 significantly inhibited proliferation, colony formation, cell migration and invasion, and induced cell apoptosis in a human HCC cell line. Knockdown of miR-2053 induced the opposite changes, increasing proliferation and migration, and reducing apoptosis. These results suggest that miR-2053 may function as a tumor suppressor in HCC. The molecular mechanisms underlying the role of miR-2053 in HCC cells remains unclear.

Previous studies have shown that the PI3K/AKT pathway is one of the most altered oncogenic pathways in tumor development, including $\mathrm{HCC}(36,37)$. The downstream effector, mTOR serves an important role in hepatitis and HCC development. Previous studies have demonstrated that AKT/mTOR serves an important role in the cell cycle of tumor cells $(38,39)$. Therefore, the phosphorylation of AKT and mTOR, as well as expression of cell cycle regulators Cyclin D1 and p70, was examined in the present study. The results demonstrated that miR-2053 overexpression significantly reduced the levels of phosphorylated AKT and mTOR, and the levels of Cyclin D1 and p70 in Huh7 cells, suggesting that miR-2053 may inhibit HCC proliferation through regulating the AKT/mTOR signaling pathway.

Wnt 3 belongs to the Wnt 1 class of ligands and stimulates the canonical Wnt/ $\beta$-catenin pathway $(18,40)$. Wnt $/ \beta$-catenin is involved in HCC progression. TRIM37 overexpression promotes cell migration and metastasis in HCC by activating Wnt/ $\beta$-catenin signaling (41). It has also been found that the downregulation of miR-200a induced epithelial-mesenchymal transition phenotypes and influenced proliferation, migration and invasion of SGC790 and U251 cells through targeting the $\beta$-catenin pathway (42). Here, miR-2503 overexpression inhibited the expression of Wnt 3 and $\beta$-catenin, and increased E-cadherin levels, downstream molecules in the Wnt signaling pathway. Taken together, these data demonstrated that miR-2053 may function as a tumor suppressor in HCC development by regulating cell migration and invasion.

In conclusion, it was demonstrated that miR-2053 overexpression inhibited Huh7 cell proliferation, colony formation, cell migration and invasion, and induced cell apoptosis. Additionally, the PI3K/AKT/ and Wnt/ $\beta$-catenin signaling pathways were regulated by miR-2053 in this HCC cell line. Therefore, miR-2053 may be a potential target for novel invasive $\mathrm{HCC}$ treatment.

\section{Acknowledgements}

Not applicable.

\section{Funding}

No funding was received.

\section{Availability of data and materials}

All data generated or analyzed during this study are included in this published article.

\section{Authors' contributions}

TS, KM, CZ, JY, JL participated in the experiments, data analysis, manuscript design and writing. All authors have read and approved this manuscript.

\section{Ethics approval and consent to participate}

Not applicable.

\section{Patient consent for publication}

Not applicable. 


\section{Competing interests}

The authors declare that they have no competing interests.

\section{References}

1. Davis GL, Dempster J, Meler JD, Orr DW, Walberg MW, Brown B Berger BD, O'Connor JK and Goldstein RM: Hepatocellular carcinoma: Management of an increasingly common problem. Proc (Bayl Univ Med Cent) 21: 266-280, 2008.

2. Di Pardo BJ, Bronson NW, Diggs BS, Thomas CR Jr, Hunter JG and Dolan JP: The global burden of esophageal cancer: A disability-adjusted life-year approach. World J Surg 40: 395-401, 2016.

3. Yang J, Yan L and Wang W: Current status of multimodal \& combination therapy for hepatocellular carcinoma. Indian J Med Res 136: 391-403, 2012

4. Thorgeirsson SS and Grisham JW: Molecular pathogenesis of human hepatocellular carcinoma. Nat Genet 31: 339-346, 2002.

5. Felekkis K, Touvana E, Stefanou C and Deltas C: MicroRNAs: A newly described class of encoded molecules that play a role in health and disease. Hippokratia 14: 236-240, 2010.

6. Wahid F, Shehzad A, Khan T and Kim YY: MicroRNAs: Synthesis, mechanism, function, and recent clinical trials Biochim Biophys Acta 1803: 1231-1243, 2010.

7. Mott JL: MicroRNAs involved in tumor suppressor and oncogene pathways: Implications for hepatobiliary neoplasia. Hepatology 50: 630-637, 2009.

8. Fendler A, Stephan C, Yousef GM and Jung K: MicroRNAs as regulators of signal transduction in urological tumors. Clin Chem 57: 954-968, 2011.

9. Roy S, Benz F, Luedde T and Roderburg C: The role of miRNAs in the regulation of inflammatory processes during hepatofibrogenesis. Hepatobiliary Surg Nutr 4: 24-33, 2015.

10. Szabo G and Bala S: MicroRNAs in liver disease. Nat Rev Gastroenterol Hepatol 10: 542-552, 2013

11. Hsu SH and Ghoshal K: MicroRNAs in liver health and disease Curr Pathobiol Rep 1: 53-62, 2013.

12. Pandey R, Jackson L, Ma G and Ahmed RP: Identification of MicroRNAs inducing adult cardiomyocyte proliferation. Circulation 130: A16314, 2014.

13. Tsai WL and Chung RT: Viral hepatocarcinogenesis Oncogene 29: 2309-2324, 2010.

14. Steelman LS, Chappell WH, Abrams SL, Kempf RC, Long J, Laidler P, Mijatovic S, Maksimovic-Ivanic D, Stivala F, Mazzarino MC, et al: Roles of the Raf/MEK/ERK and $\mathrm{PI} 3 \mathrm{~K} / \mathrm{PTEN} / \mathrm{Akt} / \mathrm{mTOR}$ pathways in controlling growth and sensitivity to therapy-implications for cancer and aging. Aging (Albany NY) 3: 192-222, 2011.

15. Galuppo R, Ramaiah D, Ponte OM and Gedaly R: Molecular therapies in hepatocellular carcinoma: What can we target? Dig Dis Sci 59: 1688-1697, 2014.

16. Vilchez V, Turcios L, Marti F and Gedaly R: Targeting Wnt/beta-catenin pathway in hepatocellular carcinoma treatment. World J Gastroenterol 22: 823-832, 2016.

17. Qu C, He, Lu X, Dong L, Zhu Y, Zhao Q, Jiang X, Chang P, Jiang $X$, Wang L, et al: Salt-inducible Kinase (SIK1) regulates $\mathrm{HCC}$ progression and $\mathrm{WNT} /$ beta-catenin activation. J Hepatol 64: 1076-1089, 2016.

18. Chu Y, Fan W, Guo W, Zhang Y, Wang L, Guo L, Duan X, Wei J and Xu G: MiR-1247-5p functions as a tumor suppressor in human hepatocellular carcinoma by targeting Wnt3. Oncol Rep 38: 343-351, 2017.

19. Yu Z, Wang Z, Li F, Yang J and Tang L: MiR-138 modulates prostate cancer cell invasion and migration via $\mathrm{Wnt} / \beta$-catenin pathway. Mol Med Rep 17: 3140-3145, 2018.

20. Tanabe H, Hayashi M, Sugimoto H, Kurimoto K, Hirabayashi S, Kanda M, Takami H, Niwa Y, Iwata N, Kobayashi D, et al: Abstract 3429: Oncogenic function of miR-23b-3p in hepatocellular carcinoma. Cancer Res 77: 3429-3429, 2017.

21. Livak KJ and Schmittgen TD: Analysis of relative gene expression data using real-time quantitative PCR and the 2(-Delta Delta C(T)) method. Methods 25: 402-408, 2001.

22. Fritz V and Fajas L: Metabolism and proliferation share common regulatory pathways in cancer cells. Oncogene 29: 4369-4377, 2010 .
23. Curran S and Murray GI: Matrix metalloproteinases in tumor invasion and metastasis. J Pathol 189: 300-308, 1999.

24. Symonds H, Krall L, Remington L, Saenz-Robles M, Lowe S, Jacks T and Van Dyke T: p53-Dependent apoptosis suppresses tumor growth and progression in vivo. Cell 78: 703-711, 1994

25. Martinou JC and Youle R: Mitochondria in apoptosis: Bcl-2 family members and mitochondrial dynamics. Dev Cell 21: 92-101, 2011

26. Gui D, Guo Y, Wang F, Liu W, Chen J, Chen Y, Huang J and Wang N: Astragaloside IV, a novel antioxidant, prevents glucose-induced podocyte apoptosis in vitro and in vivo. PLoS One 7: e39824, 2012.

27. Chen JS, Wang Q, Fu XH, Huang XH, Chen XL, Cao LQ, Chen LZ, Tan HX, Li W, Bi J and Zhang LJ: Involvement of $\mathrm{PI} 3 \mathrm{~K} / \mathrm{PTEN} / \mathrm{AKT} / \mathrm{mTOR}$ pathway in invasion and metastasis in hepatocellular carcinoma: Association with MMP-9. Hepatol Res 39: 177-186, 2010.

28. Tuo H, Wang Y, Wang L, Yao B, Li Q, Wang C, Liu Z, Han S, Yin G, Tu K and Liu Q: MiR-324-3p promotes tumor growth through targeting DACT1 and activation of Wnt/ $\beta$-catenin pathway in hepatocellular carcinoma. Oncotarget 8: 65687-65698, 2017.

29. Cao N, Mu L, Yang W, Liu L, Liang L and Zhang H: MicroRNA-298 represses hepatocellular carcinoma progression by inhibiting CTNND1-mediated Wnt/ $\beta$-catenin signaling. Biomed Pharmacother 106: 483-490, 2018.

30. Balogh J, Victor D III, Asham EH, Burroughs SG, Boktour M, Saharia A, Li X, Ghobrial RM and Monsour HP Jr: Hepatocellular carcinoma: A review. J Hepatocell Carcinoma 3: 41-53, 2016.

31. Chu R, Mo G, Duan Z, Huang M, Chang J, Li X and Liu P: MiRNAs affect the development of hepatocellular carcinoma via dysregulation of their biogenesis and expression. Cell Commun Signal 12: 45, 2014.

32. Thurnherr T, Mah WC, Lei Z, Jin Y, Rozen SG and Lee CG: Differentially expressed miRNAs in hepatocellular carcinoma target genes in the genetic information processing and metabolism pathways. Sci Rep 6: 20065, 2016.

33. Park JK, Kogure T, Nuovo GJ, Jiang J, He L, Kim JH, Phelps MA, Papenfuss TL, Croce CM, Patel T and Schmittgen TD: MiR-221 silencing blocks hepatocellular carcinoma and promotes survival. Cancer Res 71: 7608-7616, 2011.

34. Zhang JG, Shi Y, Hong DF, Song M, Huang D, Wang CY and Zhao G: MiR-148b suppresses cell proliferation and invasion in hepatocellular carcinoma by targeting WNT1/beta-catenin pathway. Sci Rep 5: 8087, 2015.

35. Liu K, Li X, Cao Y, Ge Y, Wang J and Shi B: MiR-132 inhibits cell proliferation, invasion and migration of hepatocellular carcinoma by targeting PIK3R3. Int J Oncol 47: 1585-1593, 2015.

36. Courtney KD, Corcoran RB and Engelman JA: The PI3K pathway as drug target in human cancer. J Clin Oncol 28: 1075-1083, 2010.

37. Tu K, Liu Z, Yao B, Han S and Yang W: MicroRNA-519a promotes tumor growth by targeting PTEN/PI3K/AKT signaling in hepatocellular carcinoma. Int J Oncol 48: 965-974, 2016.

38. Chang F, Lee JT, Navolanic PM, Steelman LS, Shelton JG, Blalock WL, Franklin RA and McCubrey JA: Involvement of PI3K/Akt pathway in cell cycle progression, apoptosis, and neoplastic transformation: A target for cancer chemotherapy. Leukemia 17: 590-603, 2003.

39. Gao N, Zhang Z, Jiang BH and Shi X: Role of PI3K/AKT/mTOR signaling in the cell cycle progression of human prostate cancer. Biochem Biophys Res Commun 310: 1124-1132, 2003.

40. Kim M, Lee HC, Tsedensodnom O, Hartley R, Lim YS, Yu E, Merle P and Wands JR: Functional interaction between Wnt 3 and Frizzled-7 leads to activation of the Wnt/beta-catenin signaling pathway in hepatocellular carcinoma cells. J Hepatol 48: 780-791, 2008.

41. Jiang J, Yu C, Chen M, Tian S and Sun C: Over-expression of TRIM37 promotes cell migration and metastasis in hepatocellular carcinoma by activating Wnt/beta-catenin signaling. Biochem Biophys Res Commun 464: 1120-1127, 2015.

42. Juan S, Anling Z, Zhendong S, Ma F, Pu P, Wang T, Zhang J, Kang $C$ and Zhang Q: MicroRNA-200a suppresses the Wnt $/ \beta$-catenin signaling pathway by interacting with $\beta$-catenin. Int J Oncol 40: 1162-1170, 2012. 